\title{
Criminologie
}

\section{Les enfants de parents incarcérés aux États-Unis : une analyse} qualitative

\author{
A Qualitative Analysis of Children whose Parents Are \\ Incarcerated in the US

\section{Los hijos de padres encarcelados en los Estados Unidos: un análisis cualitativo}

\section{Megan Sullivan}

Volume 52, numéro 1, printemps 2019

Les proches de personnes judiciarisées : expériences humaines et connaissances carcérales

URI : https://id.erudit.org/iderudit/1059541ar

DOI : https://doi.org/10.7202/1059541ar

Aller au sommaire du numéro

Éditeur(s)

Les Presses de l’Université de Montréal

ISSN

0316-0041 (imprimé)

1492-1367 (numérique)

Découvrir la revue

Citer cet article

Sullivan, M. (2019). Les enfants de parents incarcérés aux États-Unis : une analyse qualitative. Criminologie, 52(1), 97-117.

https://doi.org/10.7202/1059541ar
Résumé de l'article

Aux États-Unis, l'importante hausse du taux d'incarcération, le nombre imposant d'individus condamnés pour des infractions liées aux drogues et la multiplication de sentences de longue durée signifient que plus de la moitié des prisonniers sont des parents. Des recherches se sont penchées sur les conséquences de l'incarcération d'un parent sur les enfants, mais la plupart ont abordé le moment de l'incarcération et non l'expérience de ces enfants au cours de leur vie. La présente étude, une analyse qualitative se fondant sur des données empiriques et adoptant une approche développementale, examine les expériences de 35 adultes qui révèlent en quoi l'incarcération de leurs parents les a affectés. L'étude se concentre sur trois éléments. D’abord, sur le fait que les conséquences de l'incarcération d'un parent ne sont pas les mêmes pour tous les enfants et varient en fonction de la période à laquelle le parent a été incarcéré. Ensuite, le genre (l'identité sexuelle) du parent incarcéré et les répercussions économiques de l'incarcération sont des variables importantes à considérer. Enfin, la période de réinsertion sociale est plus complexe qu'on ne le reconnaît généralement. 


\title{
Les enfants de parents incarcérés aux États-Unis: une analyse qualitative
}

\author{
Megan Sullivan ${ }^{1}$ \\ Vice-doyenne, Faculté recherche et développement \\ Directrice, centre d'enseignement et d'apprentissage interdisciplinaire \\ Université de Boston \\ msullvan@bu.edu
}

Article traduit de l'anglais au français par Florence Dubois

RÉSUMÉ - Aux États-Unis, l'importante hausse du taux d'incarcération, le nombre imposant d'individus condamnés pour des infractions liées aux drogues et la multiplication de sentences de longue durée signifient que plus de la moitié des prisonniers sont des parents. Des recherches se sont penchées sur les conséquences de l'incarcération d'un parent sur les enfants, mais la plupart ont abordé le moment de l'incarcération et non l'expérience de ces enfants au cours de leur vie. La présente étude, une analyse qualitative se fondant sur des données empiriques et adoptant une approche développementale, examine les expériences de 35 adultes qui révèlent en quoi l'incarcération de leurs parents les a affectés. L'étude se concentre sur trois éléments. D'abord, sur le fait que les conséquences de l'incarcération d'un parent ne sont pas les mêmes pour tous les enfants et varient en fonction de la période à laquelle le parent a été incarcéré. Ensuite, le genre (l'identité sexuelle) du parent incarcéré et les répercussions économiques de l'incarcération sont des variables importantes à considérer. Enfin, la période de réinsertion sociale est plus complexe qu'on ne le reconnaît généralement.

MOTS CLÉs - Parents incarcérés, enfants de parents incarcérés, détenus, enfants de détenus, développement.

\section{Contexte}

Les enfants de parents incarcérés

Aux États-Unis, l'importante hausse du taux d'incarcération, le nombre imposant d'individus condamnés pour des infractions liées aux drogues

1. Division of Rhetoric, College of General Studies, Boston University, 871 Commonwealth, Boston, MA, 02215, États-Unis. 
et la multiplication de sentences de longue durée signifient que plus de la moitié des prisonniers sont des parents (Mauer, Nellis et Shirmir, 2009). Selon le premier sondage national portant sur l'incarcération de parents, plus de 700000 parents sont des détenus (Mumola, 2000). De nos jours, 2,7 millions d'enfants aux États-Unis ont un parent incarcéré, et près de 10 millions d'enfants ont connu l'incarcération d'un parent (National Resource Center on Children and Families of the Incarcerated [NRCCFI], 2014). Étant donné qu'un nombre disproportionné de personnes non blanches sont incarcérées, plus d'enfants d'origine non blanche sont touchés par cette situation: 1 enfant d'origine afro-américaine sur 9; 1 enfant d'origine latino-américaine sur 28 ; et 1 enfant d'origine européenne sur 57 ont un parent incarcéré (NRCCFI, 2014).

Les premières recherches sur les enfants de détenus aux États-Unis se sont centrées sur la relation entre les crimes des parents et le comportement délinquant des enfants (Baker, Mack, Moffitt et Mednick, 1989; Bohman, Cloninger, Sigvardsson et von Knorring, 1982; Glueck et Glueck, 1950; Loeber et Dishion, 1983; McCord, 1979; Reiss et Roth, 1993; Robins, 1978; Wilson et Hernstein, 1985). Si ces recherches ont établi un lien de causalité entre les deux, elles n'ont jamais pu montrer le mécanisme de transmission intergénérationnelle de comportements criminels (Johnston, 2006).

Dans les années 1980, le nombre de femmes incarcérées aux ÉtatsUnis a plus que triplé (Sabol, Couture et Harrison, 2007). Les chercheurs ont alors délaissé la délinquance juvénile pour se concentrer sur les familles (Adalist-Estrin, 2006; Arditti, Lambert-Shute et Jost, 2003 ; Baunauch, 1985; Bloom et Steinhart, 1993; Boswell, 2002; Costa, 2003 ; Gabel et Johnston, 1995 ; Mazza, 2002 ; Mumola, 2000; Myers, Smarsh, Amlund-Hagen et Kennon, 1999; Parke et Clarke-Stewart, 2001; Patton, 1999; Seymour et Finney, 1998; Simmons, 2000; Travis, McBride et Solomon, 2003 ; Young et Smith, 2000).

Les recherches réalisées par la suite ont relevé que l'incarcération d'un parent allait avoir des conséquences sur le développement et le bien-être de l'enfant (Boss, 2004; Hairston, 2007; Lee, Fang et Luo, 2013): cette situation mènerait à des conditions de vie instables (NRCCFI, 2014), augmenterait les risques pour ces enfants de vivre dans la pauvreté (Reed et Reed, 1997), serait associée à des sentiments de perte, à la stigmatisation, à de faibles résultats scolaires et à des risques plus élevés de dépendance (La Vigne, Davies et Brazzell, 2008), 
aurait des effets négatifs à long terme sur ces enfants (Arditti, 2012; Murray et Farrington, 2008; Poehlmann et Eddy, 2010), et pourrait nuire à leur développement cognitif et comportemental (Geller, Cooper, Garfinkel, Schwartz-Soicher et Mincy, 2012). Certains chercheurs ont constaté que les enfants de parents incarcérés tendent à avoir davantage de démêlés avec la justice criminelle que les adolescents et les adultes qui n'ont pas connu l'incarcération d'un parent (Siegel, 2011).

Ces recherches sur les familles ont permis de mieux définir le problème et de mettre en place des services pour ces jeunes (Johnston, 2006). Toutefois, la plupart de ces travaux n'étaient ni longitudinaux ni ne s'appuyaient sur des preuves empiriques. De plus, puisque ces recherches étaient principalement axées sur la période pendant laquelle le parent était incarcéré, elles n'ont pas pu prendre en compte les effets à long terme sur la vie des enfants. Dans notre étude, ma collègue et moi avons privilégié une perspective centrée sur les enfants et leur développement, considérant le fait qu'ils en sont affectés bien au-delà du moment où se produit l'incarcération (Johnson et Sullivan, 2016). Afin de comprendre comment ces enfants sont affectés par l'incarcération de leur parent au cours de leur vie, nous avons analysé le fruit de leurs réflexions à l'âge adulte.

Nous avons procédé à une analyse qualitative fondée sur des preuves empiriques en vue d'étudier les effets de l'incarcération d'un parent au cours de la vie de son enfant. Cette étude nous a permis de tirer plusieurs conclusions, mais seulement trois seront discutées dans cet article. D'abord, les conséquences de l'incarcération d'un parent ne sont pas les mêmes pour tous les enfants et varient en fonction du moment où le parent est incarcéré. Ensuite, le genre (l'identité sexuelle) du parent incarcéré et les répercussions économiques de l'incarcération sont des variables importantes à considérer. Enfin, la période de réinsertion sociale est plus complexe qu'on ne le reconnaît généralement.

\section{Méthodologie}

L'étude se fonde sur des données empiriques collectées à partir d'une méthode qualitative. Elle considère que des événements significatifs influencent les enfants au-delà du moment où ils se produisent et s'intéresse aux enfants dans leur communauté et au cours de leur vie.

Suivant l'approche développementale, le développement des enfants est influencé par leurs expériences et est le résultat des effets tant du 
soutien reçu que du non-respect subi (Sroufe, Egeland, Carlson et Collins, 2005). Une étude des risques et de l'adaptation de la naissance à l'âge adulte a été menée à partir de 1976 au Minnesota (Minnesota Study of Risk and Adaptation from Birth to Adulthood) afin d'analyser la complexité du développement des enfants. Selon le rapport, le comportement des enfants est compris en fonction de changements liés à leurs expériences. Ces jeunes ne progressaient pas uniquement en fonction de la façon dont on répondait à leurs besoins primaires. Ils étaient davantage influencés lorsque leurs besoins sociaux ou émotionnels étaient comblés ou non et par les expériences qu'ils vivaient. La même étude a également conclu que les enfants sont influencés par leur famille et par les opportunités et les difficultés rencontrées au sein de leur communauté (Sroufe et al., 2005). Lorsqu'on s'intéresse aux enfants de parents incarcérés, la perspective développementale permet de considérer les changements que ces jeunes vivent en fonction des opportunités qui se présentent à eux, et le fait que les événements vécus à cette période de leur vie peuvent avoir une influence tout au long de leur vie.

Dans le cadre de notre analyse narrative, ma collègue et moi avons sollicité des témoignages à des organismes qui œuvrent auprès des familles, avons utilisé les réseaux sociaux et avons écrit aux directions de prisons. Notre directive de départ était somme toute assez large: nous mentionnions être en train de rédiger un livre de témoignages et de réflexions d'adultes ayant eu un parent incarcéré quand ils étaient enfants, et leur avons demandé de nous faire part de leur histoire. Une fois l'intérêt confirmé, nous leur avons demandé leur adresse, leur genre, leur origine ethnique, leur âge, leur profession, s'ils avaient eu des démêlés avec la justice pénale et le statut de leur parent incarcéré. Ils pouvaient utiliser des pseudonymes et envoyer leurs témoignages écrits par courrier ou par courriel. Certains témoignages provenaient de prisons, écrits au crayon sur des feuilles de papier. Bref, nous avons demandé aux participants de raconter leur vie en tant qu'enfant de parent incarcéré.

Les répondants étaient âgés de 18 à 51 ans. Parmi eux, $60 \%$ étaient des femmes et $40 \%$ des hommes. Les Afro-Américains et les Américains d'origine européenne composaient chacun $37 \%$ de ce groupe; $15 \%$ étaient d'origine latino-américaine; $6 \%$ étaient d'origine autochtone, et $6 \%$ étaient d'origine multiethnique. Soixante-six pour cent provenaient de familles à faible revenu. Les 35 participants ont décrit 
45 parents incarcérés : $60 \%$ de ces derniers étaient des pères et $11 \%$, des mères. Si $29 \%$ de nos participants avaient deux parents incarcérés, moins de $50 \%$ d'entre eux ont rapporté que leur parent incarcéré était celui qui répondait régulièrement à leurs besoins, et près des deux tiers vivaient, au moins une partie du temps, avec l'autre parent biologique une fois le parent incarcéré. Les professions des participants: vendeurs, serveurs, enseignants, étudiants, infirmiers auxiliaires, agents immobiliers, producteurs pour la télévision, professeurs universitaires et avocats. Ces participants provenaient de tous les coins du pays, mais ils étaient plus nombreux à venir des États dont les taux d'incarcération sont plus élevés: la Californie, l'Ohio, la Pennsylvanie, le Texas et l'État de Washington. Alors que $12 \%$ n'avaient pas d'emploi au moment de leur témoignage, $9 \%$ suivaient des traitements à domicile et $6 \%$ étaient sans domicile fixe. La moitié des participants avaient eu des démêlés avec la justice (79\% d'hommes et $19 \%$ de femmes), et $30 \%$ d'entre eux étaient incarcérés au moment d'écrire leur témoignage. L'incarcération intergénérationnelle a été observée chez $33 \%$ de ceux qui n'avaient connu que l'incarcération de leur père, chez $50 \%$ de ceux qui n'avaient connu que l'incarcération de leur mère et chez $70 \%$ de ceux qui avaient connu l'incarcération de leurs deux parents (Tableau 1).

TABLEAU 1

Les participants

\begin{tabular}{|l|l|l|}
\hline \multicolumn{3}{|c|}{ Renseignements sur les participants } \\
\hline Nombre total & 35 \\
\hline Âge & $18-59$ ans \\
\hline Sexe & Féminin: $60 \%$ & Masculin: $40 \%$ \\
\hline Ménages à très faible revenu & $66 \%$ & \\
\hline
\end{tabular}

\section{Résultats}

Les 35 participants ont parlé des relations qu'ils ont entretenues enfant, adolescent et à l'âge adulte, de la manière dont ils ont été éduqués, des soins et conseils qu'ils ont reçus et de la manière dont ils ont vécu l'implication de leurs parents dans le système de justice pénale. Il ressort des témoignages que l'incarcération d'un parent n'est pas une expérience monolithique pour les enfants et qu'elle varie. Le genre du parent incarcéré et le statut économique de la famille sont aussi des 


\section{F I G U RE 1}

\section{Caractéristiques des participants et des parents incarcérés}

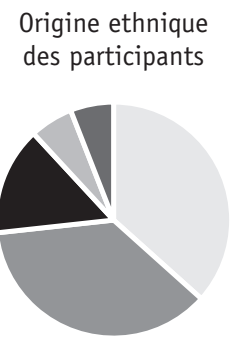
Afro-américaine Européene
Latino-américaine Autochtone
Pluriethnique

Incarcération des parents

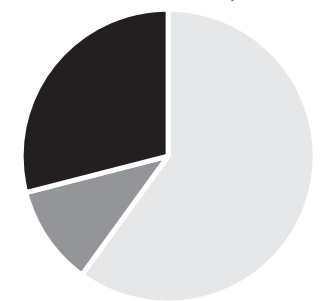

Incarcération du père

- Incarcération de la mère

- Incarcération des deux parents

Transmission intergénérationnelle de comportements criminels

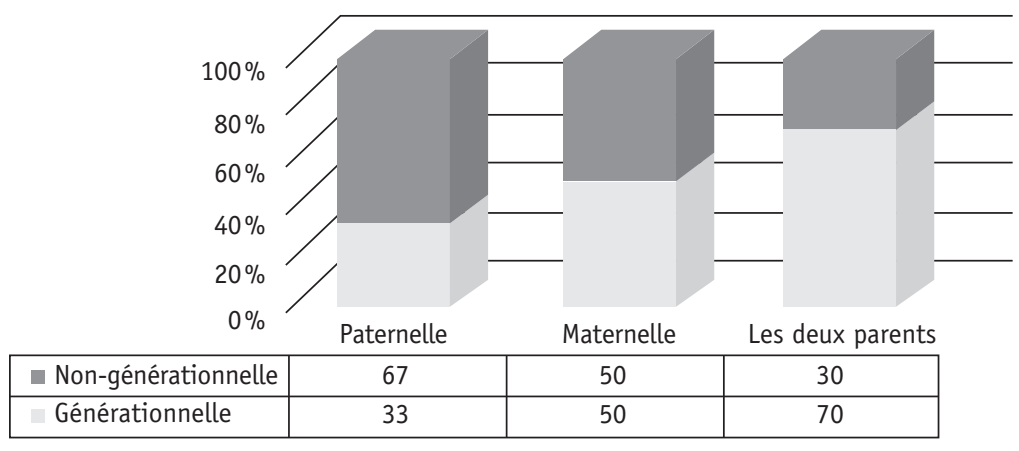

variables importantes. L'expérience qu'ils ont de la période de réinsertion sociale varie également.

L'incarcération d'un parent n'est pas une expérience monolithique pour les enfants et varie à travers les générations de prisonniers

L'expérience des enfants dont les parents sont en prison varie à travers les générations de prisonniers et en fonction des ressources alors disponibles. Une des raisons expliquant la multiplicité de ces expériences est que, d'un point de vue historique, la manière dont un pays conçoit et punit les 
crimes change au fil du temps. Les disparités sociales, la longueur des sentences et la disponibilité des services évoluent également.

L'accès des familles à des ressources aussi simples qu'un logement, de la nourriture, des services de santé et d'éducation aident à déterminer l'avenir d'un enfant. Les booms et les crises économiques, l'accès aux logements et aux services de santé et le financement public de l'éducation varient énormément au fil des décennies et même des années. Ces différences signifient des expériences encore plus diversifiées de l'incarcération d'un parent et l'on en tient souvent peu compte en étudiant la question des enfants.

Les parents de nos participants les plus âgés avaient fait de la prison avant les années 1970, à un moment où la majorité des prisonniers étaient d'origine euro-américaine (Langan, 1991) et souvent emprisonnés pour des vols. Les parents de la génération suivante, dans les années 1970, ont été condamnés au cours de la "guerre contre la drogue» et du durcissement des directives fédérales en matière de détermination de la peine. La plupart de ces parents étaient condamnés pour des délits liés à la drogue et un plus grand nombre de prisonniers étaient d'origine non blanche. Les participants les plus jeunes avaient des parents qui avaient été incarcérés durant la continuation de la «guerre contre la drogue» des années 1980 et 1990.

Que signifient donc ces différences d'époque pour les enfants? Au fur et à mesure qu'augmentait le nombre de personnes de groupes d'origines autres dans les prisons, leurs communautés se sont mises à changer. Les quartiers offraient moins de ressources et étaient marqués d'une plus grande pauvreté. Les parents incarcérés pour des délits liés à la drogue montraient des taux plus élevés de dépendance à un moment où les ressources pour la toxicomanie et l'alcoolisme n'étaient pas disponibles. La désinstitutionnalisation des années 1970 a aussi donné lieu à une diminution des services disponibles pour les gens souffrant de troubles de santé mentale. Plus d'enfants avaient alors des parents souffrant de tels troubles et abusant de substances illicites tout en étant rassemblés dans des communautés qui étaient dans l'incapacité de les aider. Depuis la modification des lois sur la détermination de la peine dans les années 1990, les sentences se sont allongées (Mauer et al., 2009), les enfants des détenus ayant alors dû passer de plus longues périodes sans leurs parents, et leur communauté sans leurs membres.

Betty (pseudonyme) est une femme afro-américaine née en 1959. Dans notre étude, elle explique n'avoir jamais connu son père, qui avait 
été incarcéré pour vol. Betty nous écrit: "[Ma mère] nous disait que c'était un homme bon qui avait volé de l'argent parce que nous étions pauvres.» Le témoignage de Betty correspond aux résultats des travaux qui expliquent qu'avant les années 1970, davantage d'individus étaient incarcérés pour des crimes liés à la propriété et au vol (Morsy et Rothstein, 2016).

La mère de Betty a élevé seule ses six enfants, au cours des années 1960, dans le sud des États-Unis. Elle n'a jamais connu son père et dit qu'il ne lui a jamais manqué. Elle nous écrit que même si «les Noirs ne pouvaient pas trouver de travail» à l'époque, sa mère travaillait en service domestique. Elle se rappelle aussi une enfance plutôt satisfaisante. Vers la fin de son témoignage, Betty explique que ses propres enfants sont sous la tutelle de l'État, qu'elle a connu des problèmes de toxicomanie et a été sans domicile fixe. Elle dit être sobre au moment où elle nous écrit et se demande si l'incarcération de son père a eu une influence sur elle. "Je ne sais pas si l'incarcération de mon père m'influence. Je ne l'ai jamais rencontré, pas une fois», explique-t-elle vers la fin de ses réflexions.

Moe-Moe, une jeune Afro-Américaine dont le père a été incarcéré plus de 20 ans après celui de Betty, est l'enfant de deux parents souffrant de toxicomanie. Son message nous laisse penser que cette dépendance est l'élément qui a le plus influencé son sort. Elle nous écrit: «Ma vie a été un enfer dès la seconde où j'ai été conçue. Ma mère a accouché prématurément parce qu'elle fumait du crack.» Abandonnée par sa mère, Moe-Moe a été élevée par son père jusqu'à ce que sa dépendance s'intensifie. Les réflexions de Moe-Moe sur cette période de sa vie montrent que les choix de son père toxicomane ont influencé la façon dont on s'occupait d'elle. Elle a été placée en foyer d'accueil après l'incarcération de son père, mais déjà à l'âge de neuf ans, elle avait traversé de lourdes épreuves.

D'abord, le père de Moe-Moe a introduit une autre toxicomane dans leur foyer, une femme dont le frère a abusé d'elle: «En plus du fait que ma belle-mère me battait et encourageait mon père dans sa toxicomanie, son frère abusait de moi.» Ensuite, à cause de la dépendance de son père, la jeune fille et ses frères et sœurs plus jeunes se sont retrouvés sans abri sécuritaire et sans source alimentaire suffisante. Moe-Moe a aussi été ostracisée. Il vaut la peine de rapporter sa réflexion:

Je me souviens de cette journée. Il n'y avait rien à manger dans la maison et ce gars nommé Chris, un ami proche de mon père, a cuisiné des haricots. 
Je n'aime même pas les haricots, mais c'est tout ce qu'on avait. Après avoir mangé, je suis sortie et je me suis assise sur le perron. Mon amie est sortie de sa maison et je lui ai demandé si elle voulait venir chez nous. Elle m'a répondu: «Ma mère ne veut pas parce que c'est une maison de drogués.»

Le père de Moe-Moe est incarcéré pour possession d'héroïne alors qu'elle a neuf ans, et elle et son frère sont placés dans des maisons d'accueil séparées. Moe-Moe passera neuf ans dans le système. Les réflexions de la jeune femme soulignent combien elle aime son père et combien il lui manque. Elle déplore son problème de toxicomanie et le fait que son incarcération l'a enlevé à eux. Mais elle aurait surtout voulu qu'il ait été «testé pour drogues au moment de sa naissance», et souhaite que les enfants n'aient pas à entrer dans le système de familles d'accueil.

Betty et Moe-Moe sont deux femmes afro-américaines dont le père a été incarcéré. Toutefois, en partie à cause de l'époque différente à laquelle cela s'est produit, leurs circonstances et leurs besoins ont été très différents. Le père de Moe-Moe a été incarcéré pour des délits de drogue et a été condamné à une sentence plus longue à cause de la «guerre contre la drogue» et aux nouvelles lois plus strictes sur les sentences (Morsy et Rothstein, 2016). La jeune fille a donc eu deux parents toxicomanes incarcérés et elle a été placée en système d'accueil. La mère de Betty n'est pas allée en prison et a dû s'occuper de la famille. Même si elle était pauvre, elle travaillait et avait donc quelques ressources. Betty n'est pas certaine des effets de l'incarcération de son père sur sa vie, alors que l'enfance de Moe-Moe a été affectée par la dépendance de ses parents et que l'incarcération de son père est la raison pour laquelle elle a été placée en famille d'accueil. À la fin de son témoignage, la jeune fille mentionne qu'elle veut que les gens sachent que «[les enfants] ne sont pas toujours bien traités dans les mains d'autres personnes». Elle considère que son triste parcours (dans le système des familles d'accueil) est directement lié à l'incarcération de son père.

\section{L'importance du genre et de la classe économique}

Certains chercheurs soutiennent que le genre du parent incarcéré est un facteur important dans le destin des enfants. Les mères incarcérées auraient davantage tendance à être le principal soutien de la famille, seraient plus en contact avec leurs enfants que les pères incarcérés et connaîtraient des conditions de vie et des expériences plus à risque 
d'affecter leur rôle parental. Les pères auraient plus tendance à être séparés de leurs enfants pour de longues périodes (Arditti, 2012; Gabel et Johnston, 1995).

Toutefois, des recherches récentes montreraient que, en ce qui a trait au comportement des enfants, les effets néfastes qu'ils subissent proviennent plus souvent des difficultés qu'ils ont vécues avant l'incarcération de leur mère plutôt que durant l'incarcération (Wildeman et Turney, 2014). Nos participants dont la mère avait été incarcérée semblaient considérer leur parcours comme plus difficile que ceux dont le père avait été incarcéré, surtout quand la mère était la personne qui offrait le soutien principal. S'il est difficile de tirer des conclusions à partir de notre échantillon, les témoignages des participants ont mis en lumière les effets du genre du parent incarcéré sur les enfants.

Les témoignages montrent que les enfants de mères incarcérés sont plus à risque de devenir des sans-abri et de vivre dans des conditions précaires après l'incarcération. Alisha, une Afro-Américaine, se rappelle être revenue chez elle, en sixième année, et avoir trouvé sa maison retournée par la police et jonchée de détritus de drogue. Sa mère n'y était plus. Alisha raconte: «Je savais que je devrais quitter l'appartement tôt ou tard... j'ai dormi dans un parc proche de l'école pour que je ne sois pas en retard le lendemain matin.» Elle s'est donc retrouvée sans abri à cause de l'incarcération de sa mère. Au début, elle changeait régulièrement de domicile, puis a fini par emménager avec un ami, puis dans un autre État pour vivre avec une sœur qu'elle connaissait à peine. Lorsque sa mère est sortie de prison, Alisha a vécu dans des foyers et des appartements avec elle. Alisha a par la suite trouvé un logement stable et a terminé un programme de formation professionnelle. Sa mère est alors incarcérée à nouveau, dans un autre État, et ne peut se présenter à la collation des grades de sa fille. S'étant trouvée à la rue précisément à cause de l'incarcération de sa mère, Alisha connaît ensuite pendant des années une instabilité de sa situation de logement.

Victoria, Afro-Américaine dont la mère a été incarcérée, décrit leur première séparation. Une analyse comportementale montrerait que cette séparation a empêché l'attachement parent-enfant.

Ma tante m'a ramenée de l'hôpital quand j'étais bébé parce que ma mère a été arrêtée et emmenée en prison après ma naissance. Elle a été incarcérée de manière intermittente après ça, jusqu'à ce que j'aie reçu mon diplôme d'études à 22 ans. La plupart des fois, elle était accusée... elle avait une dépendance à la cocaïne et à l'héroïne... Mes tantes m’ont élevée et ma 
mère apparaissait de temps en temps, mais ses visites étaient toujours courtes et se terminaient toujours dans le désastre et parfois dans le traumatisme émotionnel.

Victoria ne parle pas de sa relation avec ses tantes, mais elle considère que les visites de sa mère pendant qu'elle était sous leur responsabilité ont été perturbantes. Même après s'être juré qu'elle garderait ses distances avec sa mère, juste après avoir terminé ses études, Victoria finit par retourner chez elle parce que sa mère est victime de maltraitance physique. Cette dernière y survit, mais ne peut plus prendre soin d'ellemême. Sa fille la loge et la nourrit donc.

Plusieurs recherches montrent que la prise en charge d'un enfant est une expérience genrée, ou que les femmes ont plus tendance à s'occuper de leurs parents âgés ou malades (Revenson et al., 2016). Même si sa mère a longtemps été un élément perturbateur dans sa vie, Victoria a tout de même fini par prendre soin d'elle.

Des études relèvent que les familles sont économiquement désavantagées par l'incarcération d'un parent (Reed et Reed, 1997; Wildeman, 2009). Les réflexions de nos participants viennent appuyer cet argument, surtout si le parent participait à la vie financière de la famille. Mais les témoignages montrent également que, lorsqu'il est question d'instabilité économique, l'époque à laquelle le parent est incarcéré joue pour beaucoup.

Shari raconte qu'elle a grandi dans les années 1950 comme une «enfant juive de classe moyenne à Brooklyn, New York». Elle écrit que son père vendait des meubles et que, à ses 15 ans, il s'est fait arrêter pour détournement de fonds. Elle explique avoir eu honte du crime de son père, racontant que la situation financière précaire de sa famille était un résultat direct de son incarcération: «Les difficultés économiques n'arrêtaient pas. On a vraiment essayé de garder notre maison, mais on a perdu la voiture.» Dans le récit de Shari, sa mère semble distraite et émotionnellement affectée par le sort de son mari, mais elle parvient à garder les rênes de sa famille et à fournir hébergement et nourriture. Shari n'est donc pas arrachée à sa maison ou à son quartier.

Pamela, aussi une enfant des années 1950, d'origine euro-américaine, et fille d'un homme qui détournait de l'argent, note: «[Après l'incarcération de mon père], nous avons immédiatement sombré dans la pauvreté, et ma mère s'est mise à travailler.» Elle explique que sa mère était émotionnellement distante et négligente, et que son frère et elle en ont souffert. 
La vie était difficile pour Shari et Pamela. Leurs familles ont connu des difficultés émotionnelles et financières, mais leurs pères étaient incarcérés pour larcin, pas pour consommation de drogues. Ainsi, ces hommes ne reçurent pas de longues sentences imposées par la "guerre contre la drogue» ni par les nouvelles lois de détermination de la peine. Shari et Pamela sont devenues des femmes éduquées et ont fondé leur propre famille. Leurs réflexions montrent qu'un soutien économique aurait été utile à ce moment, leur famille en aurait bénéficié, cela aurait aidé à l'éducation parentale et à avoir des moments de repos. Mais contrairement à Alisha, Shari et Pamela ne sont pas devenues des sansabri, et n'ont donc pas eu besoin d'hébergement immédiat.

Natalie, d'origine pluriethnique, est devenue une scénariste et productrice télé prospère et a fondé sa propre famille. Plus jeune d'au moins une génération que Shari et Pamela, son histoire est importante pour plusieurs raisons. L'incarcération de la mère de Natalie a affecté financièrement et émotionnellement sa famille, en plus de la santé physique de la jeune femme. Mais puisque la famille a vécu alors avec la grandmère maternelle, Natalie et ses jeunes sœurs ne se sont pas retrouvées à la rue. Natalie n'indique pas la profession de son père, mais elle explique que sa mère était médecin avant que sa dépendance ne cause des problèmes juridiques et financiers à la famille. Elle dit aussi que ses parents étaient éduqués et l'encourageaient à bien réussir à l'école.

Natalie raconte qu'elle s'est sentie «complètement humiliée» par l'arrestation de sa mère et son histoire laisse entendre qu'elle éprouve de la colère envers la toxicomanie de sa mère, les problèmes financiers et le dysfonctionnement de leur foyer. Elle ajoute que les répercussions financières des problèmes juridiques de sa mère et de son incarcération ont été majeures. Nathalie s'exprime ainsi sur les difficultés financières de sa famille et les effets physiques et émotionnels sur elle-même:

Les soucis financiers qui ont suivi l'arrestation de ma mère ont été encore pires. En un an, nous n'avions plus de voiture ni de téléphone à la maison. Tout notre argent servait à payer les factures juridiques de ma mère et sa dépendance. Je suis devenue boulimique à l'école secondaire, sans aucun doute à cause du stress familial. J'avais des caries horribles parce que je vomissais tout le temps, mais personne ne m'amenait chez le dentiste. Je me souviens de nuits passées à me cogner littéralement la tête contre le mur pour que la douleur m'aide à m'endormir.

La famille de Nathalie aurait pu bénéficier de services sociaux tels que des traitements contre la dépendance et de la thérapie familiale. Elle 
nécessitait des soins médicaux et dentaires, et sa famille était dans une détresse financière évidente.

Cliff est né dans les années 1970 de deux parents adolescents inexpérimentés qui se sont séparés très tôt. Son témoignage fournit davantage de renseignements sur la manière dont le genre du parent et les circonstances économiques de la famille peuvent influencer le sort d'un enfant. Puisque Cliff a été l'enfant d'un parent incarcéré et est maintenant un adulte incarcéré, sa réflexion offre en outre une compréhension plus approfondie de la transmission intergénérationnelle de comportements criminels.

Sa mère et son conjoint violent et toxicomane ont élevé Cliff. L'homme que Cliff considère comme une figure paternelle était toxicomane et fréquemment incarcéré. Il écrit que la famille «n'avait parfois pas d'argent pour de la nourriture ou des articles scolaires» et que sa vie était «incroyablement instable... plusieurs déménagements, d'une maison à l'autre et d'une école à l'autre». Cliff explique: «À 15 ans, j'avais déjà quitté l'école, je consommais de la [méthamphétamine] à temps plein et j'avais souvent une arme sur moi.» Cliff a été arrêté à plusieurs reprises et nous écrit de la prison. Sa famille est pauvre et, si l'incarcération de son beau-père n'aide en rien, ils ne sont pas pauvres à cause de cela. Les réflexions de Cliff confirment les recherches réalisées sur les liens entre les problèmes économiques et l'incarcération d'un parent. Mais lui et sa famille ont connu après l'incarcération de son beau-père bien d'autres difficultés qui lui ont été encore plus nuisibles. Il aurait été bénéfique que sa famille reçoive des services sociaux et un soutien communautaire adéquat pendant son enfance, ainsi que de la thérapie pour la toxicomanie de sa mère. Si sa mère avait eu de l'aide pour l'éduquer, il aurait peut-être eu un meilleur départ dans la vie. Enfin, une analyse de la transmission intergénérationnelle de comportements criminels selon une approche comportementale permettrait de mieux comprendre le sort de Cliff.

Le Tableau 1 présente des données sur tous les participants. On observe que certaines des personnes qui nous ont écrit sont ou ont été incarcérées. Si notre étude ne détermine aucun mécanisme propre à l'incarcération intergénérationnelle, elle montre tout de même que les participants qui ont deux parents incarcérés sont plus susceptibles d'être incarcérés à leur tour. Un modèle pertinent pour comprendre ce phénomène peut être appuyé par une approche développementale. Selon Gabel et Johnston (1995), un enfant qui connaît des traumatismes, entre 
autres la séparation parent-enfant, tend à avoir une réponse émotionnelle qui, si elle n'est pas prise en compte, peut entraîner des problèmes d'adaptation. Un tel comportement peut se répéter et mener au crime. Une perspective développementale de la transmission intergénérationnelle de l'incarcération laisserait entendre que, pour certains enfants, vivre l'incarcération d'un parent ou d'autres expériences traumatisantes peut les mener à l'adoption de comportements et de modèles de comportements qui deviennent des activités criminelles ou ayant des implications avec la justice pénale. Cela semblerait plausible dans le cas de Cliff.

\section{Les histoires sur la réinsertion sociale des familles diffèrent}

Comme la recherche se penche généralement sur les adultes ou sur le moment où le parent est incarcéré plutôt que sur le parcours de vie des enfants, il est implicitement entendu que les enfants iront mieux une fois leurs parents libérés. Si c'est vrai dans certaines familles, d'autres ne trouvent aucun réconfort dans les retrouvailles lors de la réinsertion sociale. Des travaux ont commencé à examiner cette dernière situation.

Comme cela a déjà été documenté, les personnes détenues se heurtent à une pléthore de problèmes à leur sortie de prison (Travis, Solomon et Waul, 2001; Travis et Visher, 2005). Une étude récente a montré qu'au moins une de ces difficultés a une influence directe sur les enfants. Western et Smith (2017) expliquent que «la question du logement pose une contrainte matérielle importante à la relation entre les enfants et leurs parents après l'incarcération [notre traduction]». À partir de données de l'étude Boston Reentry, les auteurs ont constaté que les parents sans logement stable à leur sortie de prison sont beaucoup moins en contact avec leurs enfants. Si un parent ayant été incarcéré vit dans une situation de logement stable, il a alors plus de chances de maintenir une relation avec son enfant. Comme Wester et Smith (2017) l'expliquent, «un logement privé et stable semble être un type de ressource particulière favorisant la complicité entre parents et enfants [notre traduction]». Les réflexions d'Alisha, plus haut dans l'article, semblent appuyer cette idée.

En partie parce que les recherches tendent à favoriser le point de vue des adultes et en partie parce que le soutien des familles est associé à de meilleures expériences de réinsertion sociale (Hairston, 2002; Visher et Travis, 2003), des décideurs politiques et autres intervenants ont 
tenté d'appliquer ces méthodes au processus de réinsertion sociale (diZerega et Shapiro, 2007), mais ces efforts sont généralement vains. Le rapport de l'Urban Institute, Families and Reentry: Unpacking How Social Support Matters, rédigé par Jocelyn Fontaine, Douglas Gilchrist Scott, Megan Denver et Shelli B. Rossman (2012), analyse les tentatives d'un programme visant à faire participer les familles au processus de réinsertion sociale. Ces chercheurs ont conclu que les familles ont des expériences variables et très difficiles à cette étape. Par exemple, le niveau d'implication et de connexion émotionnelle d'une famille varie au cours de l'incarcération d'un parent, augmentant lorsqu'il sort de prison puis chutant de nouveau. Et il en irait de même pour la communication entre la personne libérée et sa famille.

Ces constats sont appuyés par les propos d'une participante à notre étude, surtout en ce qui a trait à la communication. Elle s'exprime clairement sur la question: «[Depuis sa libération], mon père et moi ne savons pas trop comment faire partie de la vie de l'un et de l'autre... Même s'il vit à 15 minutes de chez moi, ça fait des années que je ne l'ai pas vu... J'imagine qu'il est assez facile de discuter pendant deux heures lors d'une visite en prison, mais quand il faut s'efforcer de faire partie de la vie de quelqu'un, ce n'est pas aussi facile.»

\section{Conclusion}

Dans Invisible no more: Children of incarcerated parents in Latin American and the Caribbean, Saavedra, Lappado, Bango et Mello (2003) prônent l'idée d'un registre national des enfants de prisonniers. Ayant réalisé des entretiens auprès d'agents de protection de l'enfance et d'experts en justice, les auteurs ont souligné que la justice et le milieu pénal sont trop centrés sur les adultes. Leurs approches ne parviennent donc pas à prendre en compte les enfants de détenus et leurs droits. Si Saavedra et al. (2003) parlent de l'Amérique latine et des Caraïbes, leurs recherches sont pertinentes en contexte nord-américain. L'une des raisons pour lesquelles les spécialistes ne comprennent pas exactement comment un enfant est touché par l'incarcération d'un parent tient au cadre d'analyse utilisé surtout axé sur les adultes. La perspective développementale pourrait aider les chercheurs à adopter des approches centrées sur l'enfant. Une attention portée aux facteurs tels que la période de la vie à laquelle le parent est incarcéré, le genre du parent et le statut économique de la famille, ainsi que l'expérience 
variée de la réinsertion sociale chez les enfants permettrait de mieux comprendre leurs expériences.

Utiliser un modèle d'approche axé sur les enfants en considérant les enfants de parents incarcérés comme tous les autres «mineurs à risque» permettrait de les identifier tôt. Les enfants dont les parents sont impliqués dans le système de justice pénale, qu'il s'agisse d'incarcération, de libération conditionnelle, d'arrestation ou de toute autre forme de supervision, devraient être identifiés le plus tôt possible afin de recevoir les meilleurs services possible. Nous savons que les enfants qui connaissent des expériences difficiles sont plus à risque d'avoir des problèmes, dont des problèmes de développement (Horan et Widom, 2015). Si on repère ces enfants au moment où leurs parents sont impliqués dans le système de justice pénale, il sera plus facile de les aider (Turney, 2017). Toutefois, on ne saura pas ce qui serait réellement arrivé si Cliff, un de nos participants, avait été repéré tôt, au moment de l'incarcération de son père, et s'il avait été possible de lui venir en aide. De même, la vie d'Alisha serait peut-être la même si elle avait obtenu un soutien de la société, mais elle ne se serait sans doute pas retrouvée sans domicile si la police avait été formée, comme elle l'est dans certains États, à se renseigner si la personne qu'elle arrête a des enfants. Turney (2017) note qu'une majorité de chercheurs se sont intéressés aux conséquences intergénérationnelles «normales» de l'incarcération d'un parent sans tenir compte des diverses manières dont elles affectent les enfants. Cette étude mentionne que les chercheurs devraient essayer de comprendre ces conséquences.

Deux hommes ayant participé à notre étude ont mentionné qu'ils auraient souhaité être identifiés et obtenir de l'aide après l'incarcération de leur mère, voire avant. L'un d'eux explique:

Après que ma mère est allée en prison et en réhabilitation, une travailleuse sociale nous a ramenés à la même maison, dans la même pièce où nous avions trouvé notre mère [en état de surdose] neuf mois plus tôt. C'est encore douloureux de penser que la cour nous a laissés de nouveau sous la responsabilité de notre mère.

Un autre déplore:

J'ai vu ma mère être constamment arrêtée par les policiers de Milwaukee... Comme je ne pouvais pas trouver d'amour inconditionnel, en passant d'une maison à l'autre, alors que ma mère faisait des allers-retours à la prison du comté de Milwaukee, j'ai trouvé du réconfort auprès de délinquants dans la rue. 
Les mères de ces deux participants ont été incarcérées à plusieurs reprises. Il y a eu plusieurs occasions où les intervenants des établissements correctionnels et des services sociaux auraient pu s'inquiéter du sort des enfants et intervenir d'une manière appropriée. Les enjeux de telles interventions sont majeurs. Les deux participants sont devenus des adultes incarcérés.

Lorsque nous avons demandé à ces adultes de nous parler de leur expérience en tant qu'enfants de parents incarcérés, nous en avons appris davantage sur les multiples répercussions sur leur enfance et sur leur vie en général. Si nous nous occupons des enfants et étudions leur situation seulement au moment où leur parent est incarcéré, nous passons à côté de variables importantes, telles que le genre, le statut économique, la période de vie et la réinsertion sociale, pouvant avoir un effet sur les obstacles et les opportunités qui se présentent aux enfants. Une approche qualitative qui adopte une perspective développementale et demande à des adultes de réfléchir à leur expérience offre une information pertinente sur le sort des enfants avant, pendant et après l'incarcération d'un parent.

\section{Références}

Adalist-Estrin, A. (2006). Providing support to adolescent children with incarcerated parents. The Prevention Researcher, 13(2), 7-10.

Arditti, J. A. (2012). Parental incarceration and the family: Psychological and social effects of imprisonment on children, parents, and caregivers. New York, NY: New York University Press.

Arditti, J. A., Lambert-Shute, J. et Jost, K. (2003). Saturday morning at the jail: Implications of incarceration for families and children. Family Relations: Interdisciplinary Journal of Applied Family Studies, 52(3), 195-204. doi: 10.1111/j.1741-3729.2003.00195.x

Baker, L. A., Mack, W., Moffitt, T. E. et Mednick, S. A. (1989). Etiology of sex differences in criminal convictions in a Danish adoption cohort. Behavior Genetics, 19(3), 355-370.

Baunauch, P. J. (1985). Mothers in Prison. New Brunswick, NJ: Transaction Books.

Bloom, B. et Steinhart, D. (1993). Why punish the children: A reappraisal of the children of incarcerated mothers in America. San Francisco, CA: National Council on Crime \& Delinquency.

Bohman, M., Cloninger, C. R., Sigvardsson, S. et von Knorring, A. L. (1982). Predisposition to petty criminality in Swedish adoptees: I. Genetic and environmental heterogeneity. Archives of General Psychiatry, 39(11), 12331241. 
Boss, P. (2004). Ambiguous loss. Dans F. Walsh et M. McGoldrick (dir.), Living Beyond Loss (p. 237-246). New York, NY: Norton.

Boswell, G. (2002). Imprisoned fathers: The children's view. The Howard Journal of Criminal Justice, 41(1), 14-26.

Costa, R. D. (2003). Now I lay me down to sleep: A look at overnight visitation rights available to incarcerated mothers. New England Journal of Criminal and Criminal Confinement, 29(1), 67-98.

Dallaire, D. H. (2007). Incarcerated mothers and fathers: A comparison of risks for children and families. Family Relations, 56(5), 440-453. doi: 10.1111/j.1741-3729.2007.00472.x

diZerega, M. et Shapiro, C. (2007). Asking about family can enhance reentry. Corrections Today, 69(6), 58-61.

Fontaine, J., Gilchrist, D. S., Denver, M. et Rossman, S. B. (2012). Families and reentry: Unpacking how social support matters. Washington, DC: Urban Institute.

Gabel, K. et Johnston, D. (dir.). (1995). Children of incarcerated parents. New York, NY: Lexington Books.

Geller, A., Cooper, C. E., Garfinkel, I., Schwartz-Soicher, O. et Mincy, R. B. (2012). Beyond absenteeism: father incarceration and child development. Demography, 49(1), 49-76. https://doi.org/10.1007/s13524-011-0081-9.

Glueck, S. et Glueck, E. (1950). Unraveling juvenile delinquency. Cambridge, MA: Harvard University Press.

Hairston, C. F. (2002). The importance of families in prisoners' community reentry. The ICCA Journal on Community Corrections, 43(1), 11-14.

Hairston, C. F. (2007). Focus on children with incarcerated parents: An overview of the research literature. Baltimore, MD: Annie E. Casey Foundation. Repéré à http://www.aecf.org/resources/focus-on-children-with-incarceratedparents /

Horan, J. M. et Widom C. S. (2015). Cumulative childhood risk and adult functioning in abused and neglected children grown up. Developmental Psychopathology, 27(3), 927-941. doi : 10.1017/S095457941400090X.

Johnston, D. (2006). The wrong road: Efforts to understand the effects of parental crime and incarceration. Criminology \& Public Policy, 5(4), 703-719. doi : $10.1111 / j .1745-9133.2006 .00414 . x$

Johnston, D. et Sullivan, M. (dir.). (2016). Parental incarceration: personal accounts and developmental impact. New York, NY: Routledge.

Langan, P. (1991). America's soaring prison population. Science, 251(5001), 1568-1573. doi : $10.1126 /$ science. 251.5001 .1568

La Vigne, N. G., Davies, E. et Brazzell, D. (2008). Broken bonds: understanding and addressing the needs of children with incarcerated parents. Washington, DC: Urban Institute. Repéré à https://www.urban.org/ research/publication/broken-bonds-understanding-and-addressing-needschildren-incarcerated-parents

La Vigne, N. G., Visher, C. et Castro, J. (2004). Chicago prisoners' reflections on returning home. Washington, DC: Urban Institute. 
Lee, R. D., Fang, X. et Luo, F. (2013). The impact of parental incarceration and mental health of young adults. Pediatrics, 131, e1188-e1195. doi: 10.1542/ peds.2012-0627.

Loeber, R. et Dishion, T. (1983). Early predictors of male delinquency: A review. Psychological Bulletin, 94(1), 68-99.

Mauer, M., Nellis, A. et Shirmir, S. (2009). Incarcerated parents and their children: Trends 1991-2007. The Sentencing Project. Repéré à http://www.sentencing project.org/wp-content/uploads/2016/01/Incarcerated-Parents-and-TheirChildren-Trends-1991-2007.pdf.

Mazza, C. (2002). And then the world fell apart: The children of incarcerated fathers. Families in Society: The Journal of Contemporary Human Services, 83(5), 521-529.

McCord, J. (1979). Some child-bearing antecedents of criminal behavior in adult men. Journal of Personality $\mho-$ Social Psychology, 37(9), 1477-1486.

Morsy, L. et Rothstein, R. (2016). Mass incarceration and children's outcomes: Criminal justice policy is education policy. Washington, DC: Economic Policy Institute. Repéré à https://www.epi.org/publication/mass-incarceration-and-childrens-outcomes /

Mumola, C. J. (2000). Incarcerated parents $\mathcal{O}$ their children (Publication NCJ 182335). Washington, DC: Bureau of Justice Statistics. Repéré à https:// www.bjs.gov/content/pub/pdf/iptc.pdf

Murray, J. et Farrington, D. P. (2008). Parental imprisonment: Long-lasting effects on boys' internalizing problems through the life-course. Development and Psychopathology, 20(1), 273-290.

Myers, B. J., Smarsh, T. M., Amlund-Hagen, K. et Kennon, S. (1999). Children of incarcerated mothers. Journal of child and family studies, 8(1), 11-25.

National Resource Center on Children \& Families of the Incarcerated. (2014). Children and families of the incarcerated fact sheet. Camden, NJ: Rutgers University-Camden. Repéré à https://nrccfi.camden.rutgers.edu/files/nrccfifact-sheet-2014.pdf.

Parke, R. D. et Clarke-Stewart, K. A. (2001). Effects of parental incarceration on young children. Communication présentée à From prison to home: The effect of incarceration \& reentry on children, families \& communities, Washington, D.C.

Patton, W. W. (1999). Mommy's gone, daddy's in prison, now what about me? Family reunification for children of single custodial fathers in prison - Will the sins of the incarcerated fathers be inherited by their children. North Dakota Law Review, 75,179.

Poehlmann, J. et Eddy, J. M. (2010). A research and intervention agenda for children of incarcerated parents. Dans J. M. Eddy et J. Poehlmann (dir.), Children of Incarcerated Parents: A Handbook for Researchers and Practitioners (p. 319-342). Washington, DC: The Urban Institute Press.

Reed, D. et Reed, E. (1997). Children of incarcerated parents. Social Justice, 24(3), 152-169. Repéré à http://www.jstor.org/stable/29767028

Reiss, A. J. et Roth, J. A. (1993). Understanding \& Preventing Violence. Washington, DC: National Academy Press. 
Revenson, T. A., Griva, K., Luszczynska, A., Morrison, V., Panagopoulou, E., Vilchinsky, N. et Hagedoorn, M. (2016). Gender and caregiving: The costs of caregiving for women. Dans T. A. Revenson, K. Griva, A. Luszczynska, V. Morrison, E. Panagopoulou, N. Vilchinsky et M. Hagedoorn, Caregiving in the illness context (p. 48-63). Londres, Royaume-Uni: Palgrave Pivot.

Robins, L. N. (1978). Sturdy childhood predictors of adult antisocial behaviour: Replications from longitudinal studies. Psychological medicine, 8(4), 611-622.

Saavedra, E., Lappado, P., Bango, M. et Mello, F. (2013). Invisible no more. Children of incarcerated parents in Latin American and the Caribbean. Case study from: Brazil, Dominican Republic, Nicaragua \& Uruguay. Gurises Unidos. Repéré à http://nnapes.org/docs/Invisible-no-more.pdf

Sabol, W. J., Couture, H. et Harrison, P. M. (2007). Prisoners in 2006. Bureau of Justice Statistics Bulletin. U.S. Department of Justice. Repéré à https://www. bjs.gov/content/pub/pdf/p06.pdf.

Seymour, C. et Finney, H. C. (dir.). (1998). Children with parents in prison: Child welfare policy, program, and practice issues. New Brunswick, NJ: Transaction Publications.

Siegel, J. A. (2011). Disrupted childhoods: Children of women in prison. New Brunswick, NJ: Rutgers University Press.

Simmons, C. W. (2000). Children of incarcerated parents. Sacramento, CA: California Research Bureau.

Sroufe L. A., Egeland B., Carlson E. A. et Collins, W. A. (2005). Development of the person. New York, NY: Guilford Press.

Turney, K. (2017). The unequal consequences of mass incarceration for children. Demography, 54(1), 361-389. doi: 10.1007/s13524-016-0543-1.

Travis, J., McBride, E. C. et Solomon, A. (2003). Families left behind: The hidden costs of incarceration and reentry. Washington, DC: Urban Institute.

Travis, J., Solomon, A. L et Waul, M. (2001). From prison to bome: The dimensions and consequences of prisoner reentry. Washington DC: Urban Institute.

Travis, R. et Visher, C. (dir.). (2005). Prisoner reentry and crime in America. Cambridge, Royaume-Uni: University Press.

Visher, C. et Travis, J. (2003). Transitions from prison to community: Understanding individual pathways. Annual Review of Sociology, 29, 89-113. Repéré à http://www.jstor.org/stable/30036962

Western, B. et Smith, N. (2017). Formerly incarcerated parents and their children. Rapport inédit.

Wildeman, C. (2009). Parental imprisonment, the prison boom, and the concentration of childhood disadvantage. Demography, 46(2), 265-280.

Wildeman, C. et Turney, K. (2014). Positive, negative, or null? The effects of maternal incarceration on children's behavioral problems. Demography, 51(3), 1041-1068.

Wilson, J. Q. et Hernstein, R. (1985). Crime and buman nature. New York, NY: Simon \& Schuster.

Young, D, S. et Smith, C. J. (2000). When moms are incarcerated: The needs of children, mothers \& caregivers. Families in Society: The Journal of Contemporary Human Services, 81(2),130-141. 


\section{A Qualitative Analysis of Children whose Parents Are Incarcerated in the US}

ABSTRACT - In the United States, a rapid rise in the incarceration rate, the large number of people convicted for drug-related offenses, and an increasing number of longer sentences means that more than one-half of prisoners are parents. While there has been research on the impact of parental incarceration on children, most of this research examines the period of the parent's incarceration and does not address how children experience parental incarceration across the course of their lives. This study, a qualitative analysis based on empirical evidence and informed by a developmental framework, examines the written narratives of 35 adults who reflect on how their parent's incarceration affected them. The study focuses on three findings. First, the impact of parental incarceration is not the same for all children and varies according to the particular historical period when the parent is incarcerated. Second, the gender of the incarcerated parent and the economic impact of incarceration on the family are important variables. Third, the re-entry period is more complicated than is often acknowledged.

KEYWORDS - Incarcerated parents, children of incarcerated parents, prisoners, prisoners' children, development.

\section{Los hijos de padres encarcelados en los Estados Unidos: un análisis cualitativo}

RESUMEN - En los Estados Unidos, la rápida subida de la tasa de encarcelamiento, el número imponente de individuos condenados por infracciones ligadas a las drogas y la multiplicación de sentencias de larga duración significan que más de la mitad de los prisioneros son padres. Investigaciones han sido efectuadas sobre las consecuencias del encarcelamiento de un padre para sus hijos, pero la mayoría de ellas se centran en el momento mismo del encarcelamiento y no en la experiencia de estos hijos a lo largo de sus vidas. Este estudio, un análisis cualitativo basándose en datos empíricos y con un enfoque centrado en el desarrollo, examina las experiencias de 35 adultos que expresan la forma en la que el encarcelamiento de sus padres los ha afectado. El estudio se centra en tres elementos. Primero, en el hecho de que el impacto del encarcelamiento de un padre no es el mismo para todos los hijos y cambia en función del período histórico en el que los padres son encarcelados. Luego, el género (la identidad sexual) del padre encarcelado y las consecuencias económicas del encarcelamiento son también variables importantes. Finalmente, el período de la reinserción social es más complejo de lo que se reconoce generalmente.

PALABRAS CLAVE - Padres encarcelados, hijos de padres encarcelados, presos, hijos de presos, desarollo. 